\title{
Medicina geral e familiar e atitudes relativas a problemas ligados ao álcool: a importância da formação no âmbito da Escola de Medicina Geral e FAMILIAR (PRIMAVERA 2016)
}

Vânia de Oliveira, ${ }^{1-2}$ Frederico Rosário, ${ }^{3-6}$ Cristina Ribeiro ${ }^{7-8}$

\section{RESUMO}

Objetivos: Os cuidados de saúde primários (CSP) são o terreno ideal para rastreio e tratamento de doentes com Problemas Ligados ao Álcool (PLA). Este estudo pretendeu avaliar as atitudes dos participantes da Escola De MEDICINA GeRAL E FAMILIAR (PrIMAVERA 2016) relativas a PLA e comparar as atitudes dos participantes do curso de Alcoologia com os dos restantes cursos. Métodos: Foram avaliadas as atitudes dos participantes através do Short Alcohol and Alcohol Problems Perception Questionnaire (SAAPPQ). Foram investigadas as associações entre sexo, idade e local de trabalho e ainda com os resultados do SAAPPQ. Resultados: Obtiveram-se 250 questionários elegíveis. A idade média foi de $28,1 \pm 2,2$ anos; $86,2 \%$ eram do sexo feminino. A Administração Regional de Saúde (ARS) mais representada foi a de Lisboa e Vale do Tejo. Pré-curso, as atitudes dos participantes dos dois grupos foram semelhantes, exceto na Adequação e Segurança. Os participantes do curso de Alcoologia sentem-se seguros ao trabalharem com estes doentes. Contudo, apenas $65 \%$ destes pontuaram acima do ponto médio no Compromisso Terapêutico. Pós-curso objetivaram-se valores significativamente superiores em todas as subescalas para os participantes do curso de Alcoologia, tendo a totalidade pontuado acima do ponto médio na Segurança e Compromisso Terapêutico.

Conclusões: As atitudes dos participantes do curso de Alcoologia perante doentes com PLA melhoraram significativamente após a formação em todas as subescalas. Cursos que tenham em conta não só conhecimento e capacidade técnica, como outras atitudes, poderão melhorar o rastreio e tratamento de doentes com PLA. Poderá ser necessária uma atenção curricular específica nos domínios da Autoestima e Satisfação para melhorar resultados futuros.

Palavras-chave: Álcool; Atitudes; Medicina geral e familiar; SAAPPQ; Formação.

\section{INTRODUÇÃO}

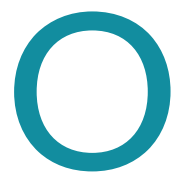

consumo de álcool ocupa atualmente uma posição relevante, não só como fator de risco de mais de 200 patologias, mas também como importante interveniente em acidentes de viação e de trabalho, violência doméstica, criminalidade, afeção de relações conjugais, entre outros. ${ }^{1-3} \mathrm{Na}$ União Europeia, $\approx 14 \%$ de todas as mortes em homens e $8 \%$ em mulheres entre os 15 e os 64 anos estão relacionadas com o álcool, ${ }^{2}$ constituindo um dos cinco principais fatores de risco de doença, incapacidade e morte no mundo. ${ }^{1}$ Traduz-se, portanto, num

1. Médica Interna de Medicina Geral e Familiar. USF São Nicolau, ACeS do Alto Ave. 2. Membro dos Corpos Sociais da Associação Portuguesa de Medicina da Adição.

3. Médico Assistente de Medicina Geral e Familiar. USF Tondela, ACeS Dão Lafões.

4. Coordenador da Equipa Projeto Investigação. ACeS Dão Lafões.

5. Coordenador da Comissão Executiva para a área de Missão Investigação em Problemas Ligados ao Álcool. Centro Académico Clínico das Beiras.

6. Consultor não permanente da Organização Mundial da Saúde para a formação e investigação em Problemas Ligados ao Álcool.

7. Professora Auxiliar. Faculdade de Medicina, Universidade de Lisboa.

8. Presidente da Associação Portuguesa de Medicina da Adição. 
peso considerável em despesas de saúde e anos potenciais de vida perdidos. ${ }^{1-2,4}$

Os cuidados de saúde primários (CSP) revelam-se o terreno ideal para o correto rastreio e tratamento destes doentes, na medida em que englobam uma importante parcela da comunidade e constituem um primeiro nível de abordagem em termos de saúde. ${ }^{5-7}$

No contexto dos CSP, as técnicas de deteção e intervenções breves consistem num conjunto de intervenções psicossociais que têm como objetivo ajudar os doentes com problemas ligados ao álcool (PLA) não só a reconhecerem o problema, como ainda motivá-los e apoiá-los a endereçá-lo. ${ }^{8}$ Em termos de duração das sessões, estas são intencionalmente breves e limitadas, por ordem a maximizar a sua viabilidade enquanto intervenção a ser utilizada ao nível dos CSP, ${ }^{9}$ podendo variar desde muito breves (cinco minutos) até sessões um pouco mais duradouras (15-30 minutos). ${ }^{10}$ Podem ser sumarizadas através dos cinco A's do aconselhamento comportametal: avaliar o consumo de álcool com uma das ferramentas de rastreio, como sejam o AUDIT, ISCA e CAGE; ${ }^{11-13}$ aconselhar ao doente para que reduza os níveis de consumo para níveis moderados; avaliar a fase do estadio de mudança em que o doente se encontra; ajudar o doente com a aquisição de motivações, técnicas de autoajuda ou apoios necessários para a mudança comportamental; e acompanhar o doente ao longo do processo de mudança. ${ }^{10}$ Os seus efeitos podem durar até cerca de 48 meses. ${ }^{14}$

No âmbito dos CSP estão já comprovadas a eficácia e o custo-efetividade de técnicas de Deteção e Intervenções Breves para o consumo excessivo de álcool (Número Necessário Tratar $=8$ ),,${ }^{7,14-20}$ baseado em evidência obtida a partir de estudos controlados aleatorizados conduzidos em vários países. ${ }^{21-22}$ Contudo, alguns autores questionam o benefício destas técnicas a nível populacional, bem como o seu impacto final ao nível da saúde pública, evidenciando falhas contempladas em estudos que demonstram a sua efetividade e sugerindo a necessidade de envolver estes doentes num contexto de cuidados longitudinais e repetidos. ${ }^{9,23}$ Ainda assim, estas técnicas continuam a ser recomendadas por entidades como a Organização Mundial da Saúde e a Direção-Geral da Saúde, ${ }^{1,15,24}$ apesar de a maioria dos médicos de família (MF) não as incluir na sua prática clínica. ${ }^{2,5-6,25-26}$
Vários autores apontam barreiras identificadas à utilização das técnicas de Deteção e Intervenções Breves. Algumas associadas a constrangimentos ambientais, nomeadamente a falta de tempo, a falta de material de aconselhamento e a falta de apoio; outras associadas a constrangimentos do médico - medo em antagonizar o doente, bem como as próprias atitudes do médico para com o doente com PLA. ${ }^{5,26-31}$ Em relação a este último aspeto, alguns autores demonstram que uma melhor atitude dos médicos em relação aos doentes com PLA está associada a um menor número de barreiras em trabalhar com estes doentes, ${ }^{32}$ podendo mesmo influenciar o número de rastreios e a diminuição do consumo de álcool por parte destes doentes. ${ }^{5,28,33}$

O estudo de Ribeiro mostra também que a maioria dos médicos em posição para realizar intervenções neste âmbito refere a falta de formação como uma das principais barreiras, resultados consistentes com os obtidos noutros

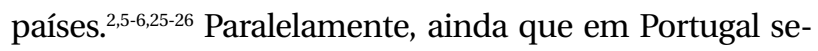
jam escassos os estudos referentes à influência da formação nas atitudes dos MF, o estudo de Família, Santos e Rosário reporta que, durante o processo de especialização, as atitudes dos internos de MGF para com os doentes com consumo excessivo de álcool mantêm-se inalteradas. ${ }^{6}$

Coloca-se, assim, em causa a relevância da inclusão de módulos de treino durante o internato capazes de melhorar as atitudes dos internos de MGF para com doentes com PLA. O presente estudo pretende verificar qual o impacto da formação em Alcoologia da EsCOLA DE Medicina Geral e Familiar (Primavera 2016) relativamente às atitudes dos internos participantes perante PLA, comparando o grupo de participantes do curso direcionado para esta temática com o grupo dos participantes dos restantes cursos, antes e após a realização dos mesmos. Pretende-se avaliar as seguintes hipóteses:

1. Diferença, pré-curso, entre as atitudes dos internos participantes do curso de Alcoologia versus outros cursos. É expectável que as atitudes dos internos participantes do curso de Alcoologia sejam melhores do que os restantes, tendo à partida um maior interesse em relação a doentes com PLA.

2. Diferença, pré versus pós-curso, entre as atitudes dos internos participantes do curso de Alcoologia versus internos dos outros cursos. Espera-se que, no final dos cursos, os internos do curso de Alcoologia tenham atitudes significativamente melhores do que os 
dos restantes cursos por, nos primeiros, ser ministrado um programa de formação com esse intuito.

\section{MÉTODOS}

População e amostra

A amostra deste estudo é constituída pelos internos participantes da Escola de Medicina Geral e Familiar (Primavera 2016) que, por sua iniciativa, se inscreveram nos cursos ministrados no evento. Todos os cursos decorreram em simultâneo, com a mesma carga horária - cerca de 33 horas.

Excluíram-se os especialistas participantes da Escola de Medicina Geral e Familiar (Primavera 2016) para garantir que possíveis diferenças nas atitudes relativas a PLA não estivessem relacionadas com um maior número de anos de prática clínica. Excluíram-se também os questionários preenchidos por participantes apenas no último dia do evento.

\section{Características da formação}

O curso de Alcoologia foi constituído por uma componente teórica e teórico-prática, incidindo em alguns temas chave - contextualização dos PLA nos CSP, epidemiologia, níveis de risco, questionários de deteção, fases de mudança, intervenções breves, álcool e gravidez, álcool e jovens, abordagem familiar, tratamento e referenciação. Todos os temas foram seguidos por um período de discussão aberta aos participantes e por diferentes formatos de exercícios práticos - aplicação de questionários de deteção, role-playing, casos clínicos, etc.

Os restantes participantes tiveram formação nos seguintes cursos: aconselhamento em CSP, atualização e treino em doenças respiratórias, cuidados paliativos, saúde dos adolescentes, atualização em diabetes.

\section{Tipo de estudo}

Estudo longitudinal observacional controlado.

\section{Colheita de dados}

O questionário foi distribuído no primeiro dia da EsCOla de Medicina Geral e Familiar (Primavera 2016) a todos os participantes, em formato papel. Este foi autopreenchido antes do início dos cursos, sendo recolhido no mesmo dia.

No final do último dia do evento, o questionário foi novamente distribuído a todos os participantes. Da mesma forma, a recolha foi feita no mesmo dia.

\section{Instrumentos de medição \\ Variáveis sociodemográficas}

Foram avaliadas as seguintes variáveis sociodemográficas: sexo; idade; categoria profissional; local de trabalho (cidade/localidade onde trabalha) que, para efeitos de estatística, foi agrupado por Administração Regional de Saúde (ARS).

\section{Medição das atitudes}

Foram avaliadas as atitudes através da resposta ao questionário Short Alcohol and Alcohol Problems Perception Questionnaire (SAAPPQ). O SAAPPQ é um instrumento validado, como demonstra a literatura. ${ }^{5,28,34}$ Utiliza uma escala de Likert de sete níveis, definidos desde «Discordo plenamente» a «Concordo plenamente», por forma a cotar 10 questões. As respostas são posteriormente somadas duas a duas, por forma a criar cinco domínios (Tabela 1): Adequação, a qual avalia até que ponto o médico acredita possuir conhecimento e habilidades suficientes para lidar com doentes com PLA; Legitimidade, a qual afere até que ponto o médico considera determinados aspetos do seu trabalho como sendo sua responsabilidade; Motivação, que determina até que ponto o médico quer trabalhar com doentes com PLA; Autoestima, que avalia até que ponto o médico entende a sua autoconfiança perante doentes com PLA; Satisfação, a qual determina até que ponto o médico se sente recompensado quando trabalha com doentes com PLA. O fator latente 'Segurança' é obtido pela soma da Adequação e Legitimidade; a soma da Motivação, Autoestima e Satisfação permite obter o fator latente 'Compromisso Terapêutico'. As perguntas referentes à Autoestima e a segunda pergunta referente à Motivação são formuladas pela negativa, pelo que as suas pontuações foram invertidas antes da análise dos dados.

\section{Consentimento informado}

O questionário contém uma área de aceitação de participação no estudo, bem como de autorização para utilização e divulgação de dados.

\section{Confidencialidade}

Foi garantida a confidencialidade e anonimato aos participantes através da utilização de um código de identificação - primeira e segunda letra do nome da mãe, primeira e segunda letra do nome do pai, três últimos dígitos do seu número do CC/BI. 
TABELA 1. Short Alcohol and Alcohol Problems Perception Questionnaire

\begin{tabular}{|c|c|c|}
\hline Fator Latente & Domínio & Pergunta \\
\hline \multirow[t]{2}{*}{ Segurança } & Adequação & $\begin{array}{l}\text { Sinto que sei o suficiente acerca das causas dos problemas ligados ao álcool para desempenhar } \\
\text { o meu papel quando lido com consumidores de bebidas alcoólicas problemáticos } \\
\text { Sinto que consigo aconselhar apropriadamente os meus doentes acerca do consumo de ácool } \\
\text { e dos seus efeitos }\end{array}$ \\
\hline & Legitimidade & $\begin{array}{l}\text { Sinto que não tenho muitos motivos de orgulho quando lido com consumidores de bebidas } \\
\text { alcoólicas } \\
\text { Tendo tudo em consideração, sinto-me inclinado a sentir insucesso quando lido com } \\
\text { consumidores de bebidas alcoólicas }\end{array}$ \\
\hline \multirow[t]{3}{*}{$\begin{array}{l}\text { Compromisso } \\
\text { Terapêutico }\end{array}$} & Motivação & $\begin{array}{l}\text { Quero trabalhar com consumidores de bebidas alcoólicas } \\
\text { Pessimismo é a atitude mais realista a adotar perante consumidores de bebidas alcoólicas } \\
\text { problemáticos }\end{array}$ \\
\hline & Autoestima & $\begin{array}{l}\text { Sinto que tenho o direito de questionar os doentes acerca dos seus hábitos alcoólicos, quando } \\
\text { necessário } \\
\text { Sinto que os meus doentes acreditam que tenho o direito de os questionar sobre os seus } \\
\text { hábitos alcoólicos quando necessário }\end{array}$ \\
\hline & Satisfação & $\begin{array}{l}\text { Em geral, é gratificante trabalhar com consumidores de bebidas alcoólicas } \\
\text { Em geral, gosto de trabalhar com consumidores de bebidas alcoólicas problemáticos }\end{array}$ \\
\hline
\end{tabular}

\section{Aprovações éticas}

O protocolo do estudo foi aprovado pela Comissão de Ética do Centro Académico de Medicina de Lisboa.

\section{Análise de dados}

Após a recolha dos questionários em formato físico foi criada uma base de dados informática em Statistical Package for the Social Sciences (SPSS), a partir da qual foi feita a caracterização dos indivíduos estudados, avaliando a idade média com os respetivos desvios-padrão, distribuição de frequência do sexo e Administração Regional de Saúde (ARS) na qual trabalha.

As respostas ao SAAPPQ foram tratadas de modo a avaliar as cinco dimensões - Adequação, Legitimidade, Motivação, Autoestima, Satisfação -, assim como os fatores latentes 'Segurança' e 'Compromisso Terapêutico' na população total. Estas variáveis foram descritas pré e pós-curso para cada um dos grupos considerados: participantes do curso de Alcoologia e dos restantes cursos.

A associação entre as variáveis nominais foi testada recorrendo ao teste exacto de Fisher. Diferenças entre a idade de acordo com o sexo foram testadas com o teste $t$ de Student para amostras independentes. Associações entre atitudes e sexo foram testadas com o teste de Mann-Whitney. As atitudes entre o grupo dos participantes do curso de Alcoologia e o grupo dos restantes cursos foram comparadas pré e pós-curso com o teste de Mann-Whitney. Um valor $P<0,05$ foi usado como ponto de corte para nível de significância.

\section{RESULTADOS}

\section{Características da amostra}

A taxa de resposta foi de 83,5\%. Foram obtidos 272 questionários no total dos dois dias, dos quais se excluiram 22 $(8,1 \%)$ por cumprirem critérios de exclusão-10 por serem preenchidos por especialistas e 12 por serem preenchidos apenas no último dia do evento. Dos 250 questionários elegíveis, 18 corresponderam a participantes que não responderam ao questionário pós-formação (Figura 1).

A amostra final $(n=116)$ tinha uma idade média de $28,1 \pm 2,2$ anos, com uma idade mínima de 25 anos e máxima de 38 anos. A maioria dos participantes era do sexo feminino $(86,2 \%)$. As ARS mais representadas foram Lisboa e Vale do Tejo (36,5\%), seguida por Norte $(28,7 \%)$ e Centro $(27,0 \%)$. Não foram encontradas diferenças significativas entre os participantes do curso de Alcoologia e os dos outros cursos quanto ao sexo, idade e ARS (Tabela 2). 


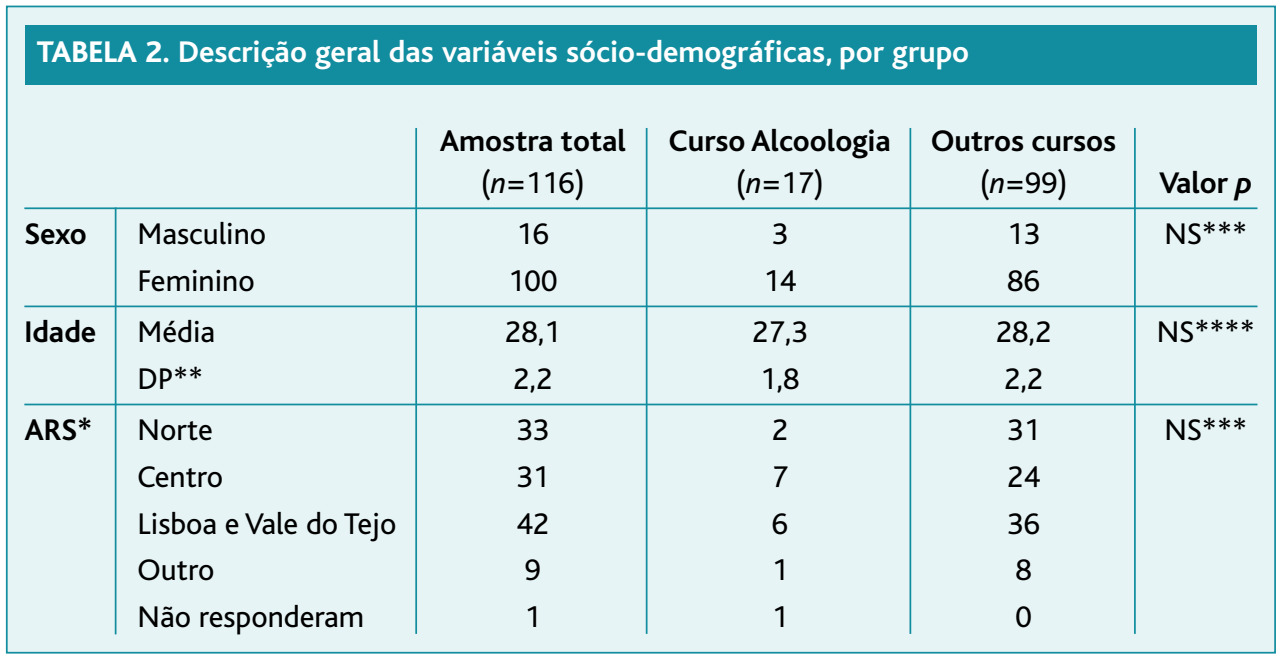

* ARS = Administração Regional de Saúde

** DP $=$ Desvio-padrão

*** Teste exacto de Fisher; NS = Não significativo

**** Teste $t$ de Student para amostras independentes; NS = Não significativo

Alcoologia. A totalidade dos internos do curso de Alcoologia pontuou acima do ponto médio na Segurança, revelando sentirem como parte do seu trabalho a gestão de doentes com PLA, para além de crerem ter conhecimento e capacidade para tal. É também evidente uma melhoria no Compromisso Terapêutico, em particular no que concerne à sua vontade em trabalhar com estes doentes.

\section{DISCUSSÃO}

Este estudo permite

Atitudes dos médicos de família relativamente a doentes com Problemas Ligados ao Álcool

\section{Resultados pré-curso}

Excetuando-se a subescala Adequação e, consequentemente, o fator latente Segurança, as atitudes dos participantes de ambos os grupos foram semelhantes (Tabela 3).

Os internos do curso de Alcoologia consideravam ser sua responsabilidade lidar com doentes com PLA, bem como ter o conhecimento e capacidade necessários para tal, com $80,0 \%$ a apresentarem valores acima do ponto médio. Apesar de motivados para esta tarefa, estes profissionais expressaram neutralidade quanto à sua autoestima em trabalharem com doentes com PLA, bem como no sentimento de recompensa quando o fazem. Ainda assim, a maioria dos internos (65\%) pontou acima do ponto médio na subescala Compromisso Terapêutico.

Paralelamente, os participantes dos restantes cursos revelam sentirem-se mais aptos no aconselhamento destes doentes, o que se traduz numa maior Segurança no desempenho das suas tarefas.

\section{Resultados pós-curso}

Pós-curso objetivam-se valores significativamente superiores em todas as subescalas avaliáveis pelo SAAPPQ para o grupo dos participantes do curso de aferir que, na baseline, as atitudes dos participantes dos dois grupos (curso de Alcoologia versus restantes cursos) são semelhantes em todas as subescalas avaliadas, com exceção da Adequação e do fator latente Segurança; após receberem a formação, os participantes do curso de Alcoologia melhoraram significativamente as suas atitudes em todas as subescalas avaliadas no SAAPPQ.

$\mathrm{Na}$ baseline as atitudes dos internos de ambos os grupos revelaram-se semelhantes em todas as subescalas, exceto na Adequação e fator latente Segurança. Tendo em conta que os participantes optaram voluntariamente pelos cursos frequentados, seria expectável que aqueles que escolheram o curso de Alcoologia tivessem um maior interesse em relação à temática do álcool e, consequentemente, atitudes mais positivas. Uma possível justificação para tal não ocorrer, em particular ao nível da Adequação, poderá prender-se com o facto dos participantes do curso de Alcoologia terem apostado nesta formação por sentirem necessidade de melhorar os seus conhecimentos e capacidades em lidar com doentes com PLA. Por outro lado, é possível que a autoperceção dos participantes dos restantes cursos não corresponda aos seus conhecimentos reais, na medida em que os médicos poderão mostrar relutância em admitir ou identificar a sua própria falha nestas circunstâncias, podendo ter sobrestimado estes 
valores. ${ }^{35}$ Ainda na baseline, a vontade em trabalhar com doentes com PLA e o sentimento de recompensa obtido pelos participantes do curso de Alcoologia parecem não acompanhar a segurança manifestada no desempenho das suas funções. Estes resultados são consistentes com os obtidos em estudos anteriores, ${ }^{5-6,26-27}$ sendo comum os MF não só acreditarem ter conhecimento para tal, como considerarem ser sua responsabilidade lidarem com estes doentes, ainda que revelem pouca autoestima e satisfação.

Quanto ao póscurso, este estudo permite concluir que os participantes do curso de Alcoologia melhoraram signifi-

TABELA 3. Valores do SAAPPQ por curso frequentado, pré e pós-curso

\begin{tabular}{|c|c|c|c|c|c|}
\hline \multicolumn{2}{|l|}{ SAAPPQ* } & \multicolumn{2}{|c|}{ Pré-curso } & \multicolumn{2}{|c|}{ Pós-curso } \\
\hline & & $\begin{array}{c}\text { Alcoologia } \\
7,5(1,8)\end{array}$ & $\begin{array}{c}\text { Outros } \\
9,0(2,0)\end{array}$ & $\begin{array}{c}\text { Alcoologia } \\
11,0(1,3)\end{array}$ & $\begin{array}{c}\text { Outros } \\
8,7(2,0)\end{array}$ \\
\hline Adequação & $\begin{array}{l}\text { Média }+(D P) \\
\% \text { acima do ponto médio** } \\
\text { Valor } p\end{array}$ & $\begin{array}{l}7,5(1,8) \\
35,0 \\
0,00\end{array}$ & $\begin{array}{l}9,0(2,0) \\
61,4 \\
* * *\end{array}$ & $\begin{array}{c}11,0(1,3) \\
100,0 \\
0,000 * * * *\end{array}$ & $\begin{array}{c}8,7(2,0) \\
54,5 \\
0,015^{* * * *}\end{array}$ \\
\hline Autoestima & $\begin{array}{l}\text { Média }+ \text { (DP) } \\
\% \text { acima do ponto médio** } \\
\text { Valor } p\end{array}$ & $\begin{array}{l}7,7(1,8) \\
30,0 \\
\quad N S^{*}\end{array}$ & $\begin{array}{c}8,3(2,0) \\
41,2 \\
\end{array}$ & $\begin{array}{c}8,9(1,6) \\
64,7 \\
0,012^{* * * *}\end{array}$ & $\begin{array}{c}8,6(2,2) \\
50,5 \\
\text { NS**** }\end{array}$ \\
\hline Motivação & $\begin{array}{l}\text { Média }+ \text { (DP) } \\
\% \text { acima do ponto médio** } \\
\text { Valor } p\end{array}$ & $\begin{array}{l}10,0(1,7) \\
80,0 \\
N S^{*}\end{array}$ & $\begin{array}{l}9,8(2,0) \\
74,1 \\
*\end{array}$ & $\begin{array}{c}11,6(1,3) \\
100,0 \\
0,002^{* * * *}\end{array}$ & $\begin{array}{c}9,5(1,7) \\
78,8 \\
\text { NS**** }\end{array}$ \\
\hline Legitimidade & $\begin{array}{l}\text { Média }+ \text { (DP) } \\
\% \text { acima do ponto médio** } \\
\text { Valor } p\end{array}$ & $\begin{array}{l}11,2(1,8) \\
95,0 \\
N S^{*}\end{array}$ & $\begin{array}{l}11,4(1,7) \\
93,9 \\
\end{array}$ & $\begin{array}{c}12,1(1,5) \\
100,0 \\
0,026^{* * * *}\end{array}$ & $\begin{array}{c}10,7(1,8) \\
89,9 \\
0,000 * * * *\end{array}$ \\
\hline Satisfação & $\begin{array}{l}\text { Média }+(D P) \\
\% \text { acima do ponto médio** } \\
\text { Valor } p\end{array}$ & $\begin{array}{l}7,8(2,2) \\
25,0 \\
\quad N S^{*}\end{array}$ & $\begin{array}{l}7,1(2,0) \\
18,4 \\
*\end{array}$ & $\begin{array}{c}8,9(1,0) \\
64,7 \\
0,004^{* * * *}\end{array}$ & $\begin{array}{c}7,3(1,8) \\
19,4 \\
0,022^{* * * *}\end{array}$ \\
\hline Segurança & $\begin{array}{l}\text { Média }+(D P) \\
\% \text { acima do ponto médio** } \\
\text { Valor } p\end{array}$ & $\begin{array}{l}18,7(2,6) \\
80,0 \\
0,007\end{array}$ & $\begin{array}{l}20,4(2,8) \\
52,6 \\
* * *\end{array}$ & $\begin{array}{c}23,2(1,9) \\
100,0 \\
0,000 * * * *\end{array}$ & $\begin{array}{c}19,4(2,9) \\
83,8 \\
0,000 * * * *\end{array}$ \\
\hline $\begin{array}{l}\text { Compromisso } \\
\text { Terapêutico }\end{array}$ & $\begin{array}{l}\text { Média + (DP) } \\
\% \text { acima do ponto médio** } \\
\text { Valor } p\end{array}$ & $\begin{array}{c}25,5(4,5) \\
65,0\end{array}$ & $\begin{array}{c}25,3(4,6) \\
56,2\end{array}$ & $\begin{array}{c}29,5(3,0) \\
100,0 \\
0,001 \mathrm{~d}\end{array}$ & $\begin{array}{c}25,4(4,2) \\
57,1 \\
\text { NS }\end{array}$ \\
\hline
\end{tabular}

* SAAPPQ = Short Alcohol and Alcohol Problem Perception Questionnaire

** Os pontos médios são os seguintes: Adequação, Autoestima, Motivação, Legitimidade = 8; Segurança = 16; Compromisso Terapêutico $=24$

*** Teste U de Mann-Whitney de amostras independentes, distribuição entre os 2 grupos; NS = não significativo

**** Teste de Wilcoxon de amostras relacionadas, diferença pré versus pós-curso dentro de cada grupo; NS = não significativo cativamente as suas

atitudes em todas as subescalas avaliadas. Estes resultados enquadram-se com o expectável, tendo em conta que se prevê que a aquisição de conhecimentos no contexto de programas de formação com as caraterísticas deste potencie melhorias significativas nas atitudes dos MF para com doentes com consumos de risco ou nocivos de álcool.

A Adequação foi a subescala cujo resultado evoluiu de uma forma mais positiva, sendo que a totalidade dos internos pontuou acima do ponto médio após a formação. Estes resultados demonstram que, através da aquisição das competências transmitidas por cursos como este, os internos se sentem suficientemente capazes e com conhecimento para lidarem com doentes com PLA. Da mesma forma, todos os internos pontuaram acima do ponto médio na subescala Legitimidade, revelando sentirem-se responsáveis pela gestão destes doentes. Estas subescalas permitem aferir uma melhoria significativa da Segurança dos participantes, resultados consistentes com os obtidos noutros estudos, ${ }^{5,28}$ o que reitera a importância da instrução na construção do profissional. Também no Compromisso Terapêutico foram obtidos resultados evolutivos favoráveis, com uma melhoria mais significativa na subescala 
Autoestima. Objetivam-se profissionais mais confiantes nas suas capacidades e com mais motivação após a formação, o que é corroborado por outros estudos. ${ }^{5,28}$

Vários autores evidenciam que a falta de treino dos profissionais constitui uma importante barreira no combate ao álcool, ${ }^{2,5-6}$ o que se traduz não só numa ineficaz identificação destes doentes como também numa insuficiente intervenção. ${ }^{5-6}$ São flagrantes alguns obstáculos e limitações à abordagem desta problemática por parte destes profissionais, ${ }^{2,5-6} \mathrm{de}$ tal forma que as atitudes dos internos perante doentes com PLA mantêm-se inalteradas ao longo do internato. ${ }^{6}$ Assim, de acordo com os resultados obtidos neste estudo, coloca-se como hipótese que uma formação que tenha em conta não só o conhecimento e capacidade técnica, como também as atitudes dos MF, seja passível de aumentar a Segurança e Compromisso Terapêutico para com doentes com PLA. .-6, $18^{-18}$

Na componente 'conhecimento', parece relevante a abordagem da informação existente acerca dos variados mecanismos e danos provocados pelo álcool, nas diferentes esferas do doente - patologias, afeção da componente relacional, profissional, etc. -, bem como em casos específicos - adolescência, gravidez, etc. Os MF devem ainda ser informados quanto à evidência existente acerca de programas de prevenção, rastreio e tratamento de doentes com PLA.

Quanto à 'capacidade técnica' deverão indicar-se instrumentos de triagem de consumos de álcool validados, como o AUDIT, ${ }^{11}$ ISCA $^{12}$ e CAGE. ${ }^{13}$ É ainda útil a abordagem de técnicas de Deteção e Intervenções Breves, cuja eficácia e custo-efetividade se apresenta já comprovada (Número Necessário Tratar $=8$ ).$^{2,14-15}$ Estas técnicas têm uma duração aproximada de 15 minutos e são acompanhadas por material escrito para o doente, sendo que os seus efeitos podem durar até 48 meses. ${ }^{14} \mathrm{~Pa}-$ ralelamente, os MF devem ser capazes de identificar situações que necessitem de referenciação para outros níveis de abordagem terapêutica.

Por fim, as 'atitudes' para com doentes com PLA podem ser abordadas através de técnicas de role-playing que simulem situações reais da prática clínica e que permitam trabalhar as diferentes esferas da postura do MF perante doentes com PLA. A utilização de questionários como o SAAPPQ poderá ser útil na autoavaliação dos profissionais para com esta temática, permitindo compreender quais os aspetos cuja abordagem seria mais benéfica. De acordo com os resultados pós-curso obtidos neste estudo, os MF apresentam maior Segurança do que Compromisso Terapêutico para lidarem com doentes com PLA, particularmente nos domínios da Autoestima e Satisfação. Em formações futuras, poderá ser necessária uma atenção curricular específica nestas áreas a fim de obter resultados mais positivos na prática clínica.

Para que as taxas de deteção e tratamento de doentes com PLA atinjam níveis cada vez mais satisfatórios seria importante abordar as atitudes dos $\mathrm{MF}^{5-6} \mathrm{a}$ fim de aumentar a sua segurança, empenho e dedicação, tornando esta tarefa mais gratificante e satisfatória para ambas as partes. Como demonstrado através do curso de Alcoologia da Escola de Medicina Geral e Familiar Primavera 2016, tal formação é passível de ser lecionada em cerca de 33 horas. Estudos adicionais são necessários para compreender a relação entre o corrente treino médico em Alcoologia fornecido durante o internato e as atitudes dos MF, bem como para aferir qual o melhor enquadramento temporal desta formação no atual currículo médico.

Identificam-se algumas limitações presentes neste estudo. A amostra utilizada poderá não ser representativa de todos os internos de MGF do país, tendo em conta que os participantes deste evento representam internos com interesse em adquirir mais conhecimentos e competências e com disponibilidade financeira para o conseguir. Por outro lado, este estudo não foi baseado numa amostra aleatorizada, uma vez que os participantes foram distribuídos pelos cursos de acordo com as suas escolhas pessoais. Contudo, à exceção da escala Adequação, os participantes revelaram-se homogéneos em todas as variáveis estudadas. Não foram ainda aferidas as escolas médicas frequentadas por todos os participantes e respetivos currículos, sendo necessários estudos adicionais para tirar conclusões quanto à relação entre diferentes programas de formação e as atitudes dos MF para com doentes com PLA. Admite-se também que o próprio preenchimento deste questionário tenha influenciado as opiniões dos participantes.

\section{CONCLUSÕES}

As atitudes dos participantes do curso específico de Alcoologia perante doentes com PLA melhoraram significativamente após a formação em todas as subescalas avaliáveis pelo SAAPPQ. Cursos que tenham em 
conta não só o conhecimento e capacidade técnica, como também as restantes atitudes dos MF, poderão ser importantes para melhorar o rastreio e tratamento de doentes com consumos de risco e nocivos de álcool. Em formações futuras, poderá ser necessária uma atenção curricular específica nos domínios da Autoestima e Satisfação a fim de obter resultados mais positivos.

\section{REFERÊNCIAS BIBLIOGRÁFICAS}

1. Poznyak V, Rekve D, editors. Global status report on alcohol and health 2014. Geneva:World Health Organization; 2014. ISBN 9789241564755

2. Anderson P, Wojnar M, Jakubczyk A, Gual A, Reynolds J, Segura L, et al. Managing alcohol problems in general practice in Europe: results from the European ODHIN survey of general practitioners. Alcohol Alcohol. 2014;49(5):531-9.

3. Balsa C, Vital C, Urbano C. III Inquérito ao consumo de substâncias psicoativas na população geral: Portugal 2012. Lisboa: Serviço de Intervenção nos Comportamentos Aditivos e nas Dependências; 2014. ISBN 9789729345876

4. Serviço de Intervenção nos Comportamentos Aditivos e nas Dependências. Relatório anual 2014: a situação do país em matéria de álcool. Lisboa: SICAD; 2015.

5. Ribeiro C. A medicina geral e familiar e a abordagem do consumo de álcool: detecção e intervenções breves no âmbito dos cuidados de saúde primários [The family medicine approach to alcohol consumption detection and brief interventions in primary health care]. Acta Med Port. 2011;24 Suppl 2:355-68. Portuguese

6. Santos G, Rosário F. Attitudes of family medicine residents towards patients with alcohol-related problems. Rev Port Med Geral e Fam. 2015; 31(6):376-82.

7. O'Donnell A, Anderson P, Newbury-Birch D, Schulte B, Schmidt C, Reimer $J$, et al. The impact of brief alcohol interventions in primary healthcare: a systematic review of reviews. Alcohol Alcohol. 2014;49(1):6678.

8. European Monitoring Centre for Drugs and Drug Addiction. Emergency department-based brief interventions for individuals with substance-related problems: a review of effectiveness. Luxembourg; Publications Office of the European Union; 2016. ISBN 9789291688364

9. Glass JE, Andréasson S, Bradley KA, Finn SW, Williams EC, Bakshi AS, et al. Rethinking alcohol interventions in health care: a thematic meeting of the International Network on Brief Interventions for Alcohol \& Other Drugs (INEBRIA). Addict Sci Clin Pract. 2017;12(1):14.

10. Anderson P, Gual A, Colom J. Alcohol and primary health care: clinical guidelines on identification and brief interventions [Internet]. Barcelona: Department of Health of the Government of Catalonia; 2005. Available from: http://inebria.net/wp-content/uploads/2017/09/cg_1.pdf

11. Babor T, Higgins-Biddle JC, Saunders JB, Monteiro MG. The alcohol use disorders identification test: guidelines for use in primary care [Internet]. 2nd ed. Geneva:World Health Organization; 2001. Available from: https://apps.who.int/iris/bitstream/handle/10665/67205/WHO_MSD_ MSB_01.6a.pdf?sequence $=1$

12. Gual A, Contel M, Segura L, Ribas A, Colom J. El ISCA (Interrogatorio Sistematizado de Consumos Alcohólicos): un nuevo instrumento para la identificación prematura de bebedores de riesgo [The ISCA (Systematic Interview of Alcohol Consumption), a new instrument to detect risky drinking]. Med Clin (Barc). 2001;117(18):685-9. Spanish

13. Ewing JA. Detecting alcoholism: the CAGE questionnaire. JAMA. 1984;252(14):1905-7.

14. Bertholet N, Daeppen JB, Wietlisbach V, Fleming M, Burnand B. Reduction of alcohol consumption by brief alcohol intervention in primary care: systematic review and meta-analysis. Arch Intern Med. 2005; 165(9):986-95.

15. World Health Organization. Evidence for the effectiveness and costeffectiveness of interventions to reduce alcohol-related harm. Geneva:WHO; 2009. ISBN 9789289041751

16. Ballesteros J, Duffy JC, Querejeta I, Ariño J, González-Pinto A. Efficacy of brief interventions for hazardous drinkers in primary care: systematic review and meta-analyses. Alcohol Clin Exp Res. 2004;28(4):60818.

17. Purshouse RC, Brennan A, Rafia R, Latimer NR, Archer RJ, Angus CR, et al. Modelling the cost-effectiveness of alcohol screening and brief interventions in primary care in England. Alcohol Alcohol. 2013;48(2):180-8.

18. Kaner EF, Beyer FR, Muirhead C, Campbell F, Pienaar ED, Bertholet N, Daeppen JB, et al. Effectiveness of brief alcohol interventions in primary care populations (review). Cochrane Database Syst Rev. 2018;2: CD004148.

19. Cuijpers P, Riper H, Lemmers L. The effects on mortality of brief interventions for problem drinking: a meta-analysis. Addiction. 2004;99(7):839-45.

20. Solberg LI, Maciosek MV, Edwards NM. Primary care intervention to reduce alcohol misuse ranking its health impact and cost effectiveness. Am J Prev Med. 2008;34(2):143-52.

21. Jonas DE, Garbutt JC, Amick HR, Brown JM, Brownley KA, Council CL, et al. Behavioral counseling after screening for alcohol misuse in primary care: a systematic review and meta-analysis for the U.S. Preventive Services Task Force. Ann Intern Med. 2012;157(9):645-54.

22. Angus C, Thomas C, Anderson P, Meier PS, Brennan A. Estimating the cost-effectiveness of brief interventions for heavy drinking in primary health care across Europe. Eur J Public Health. 2017;27(2):345-51.

23. McCambridge J, Saitz R. Rethinking brief interventions for alcohol in general practice. BMJ. 2017;356:j116.

24. Direção-Geral da Saúde. Deteção precoce e intervenção breve no consumo excessivo de álcool: norma n. ${ }^{\circ}$ 30/2012, de 28/12/2012, atualizada em 18/12/2014. Lisboa: DGS; 2014.

25. Hilbink M, Voerman G, van Beurden I, Penninx B, Laurant M. A randomized controlled trial of a tailored primary care program to reverse excessive alcohol consumption. J Am Board Fam Med. 2012;25(5):71222.

26. Geirsson M, Bendtsen P, Spak F. Attitudes of Swedish general practitioners and nurses to working with lifestyle change, with special reference to alcohol consumption. Alcohol Alcohol. 2005;40(5):388-93.

27. Wilson GB, Lock CA, Heather N, Cassidy P, Christie MM, Kaner EF. Intervention against excessive alcohol consumption in primary health care: a survey of GPs' attitudes and practices in England 10 years on. Alcohol Alcohol. 2011;46(5):570-7.

28. Anderson P, Kaner E, Wutzke S, Funk M, Heather N, Wensing M, et al. Attitudes and managing alcohol problems in general practice: an inte- 
raction analysis based on findings from a WHO collaborative study. Alcohol Alcohol. 2004;39(4):351-6.

29. Johnson M, Jackson R, Guillaume L, Meier P, Goyder E. Barriers and facilitators to implementing screening and brief intervention for alcohol misuse: a systematic review of qualitative evidence. J Public Health. 2011;33(3):412-21.

30. Nilsen P, Aalto M, Bendtsen P, Seppä K. Effectiveness of strategies to implement brief alcohol intervention in primary healthcare: a systematic review. Scand J Prim Health Care. 2006;24(1):5-15.

31. Rosário F, Santos MI, Angus K, Pas L, Fitzgerald N. Factors influencing the implementation of screening and brief interventions for alcohol use in primary care practices: a systematic review protocol. Acta Med Port. 2018;31(1):45-50.

32. Rosário F, Wojnar M, Ribeiro C. Differences between groups of family physicians with different attitudes towards at-risk drinkers: a post hoc study of the ODHIN survey in Portugal. Int J Family Med. 2016;2016:3635907.

33. O'Rourke M, Richardson LD, Wilets I, D'Onofrio G. Alcohol-related problems: emergency physicians' current practice and attitudes. J Emerg Med. 2006;30(3):263-8.

34. Anderson P, Clement S. The AAPPQ revisited: the measurement of general practitioners' attitudes to alcohol problems. $\mathrm{Br} J$ Addict. 1987;82(7):753-9.

35. Miller NS, Sheppard LM, Colenda CC, Magen J.Why physicians are unprepared to treat patients who have alcohol- and drug-related disor- ders. Acad Med. 2001;76(5):410-8.

36. Keurhorst MN, Anderson P, Spak F, Bendtsen P, Segura L, Colom J, et al. Implementing training and support, financial reimbursement, and referral to an internet-based brief advice program to improve the early identification of hazardous and harmful alcohol consumption in primary care (ODHIN): study protocol for a cluster randomized factorial trial. Implement Sci. 2013;8:11.

\section{CONFLITO DE INTERESSES}

Os autores declaram não ter quaisquer conflitos de interesse.

\section{FINANCIAMENTO}

Este estudo não foi apoiado por qualquer subsídio ou bolsa.

\section{COMISSÃO DE ÉTICA}

Estudo realizado após parecer favorável do Centro Académico de Medicina de Lisboa.

\section{ENDEREÇO PARA CORRESPONDÊNCIA \\ Vânia de Oliveira \\ E-mail: vania.oliveira@campus.ul.pt \\ https://orcid.org/0000-0002-4992-0583}

Recebido em 16-11-2019

Aceite para publicação em 05-10-2020

\section{ABSTRACT}

\section{GENERAL PRACTICE AND ATTITUDES TOWARDS PATIENTS WITH ALCOHOL-RELATED PROBLEMS: THE IMPORTANCE OF TRAINING WITHIN THE SCOPE OF SPRING 2016 General PRACTICE SCHOOL}

Introduction: Primary health care represents the ideal setting for screening and treatment of patients with Alcohol-Related Problems. This study aims to assess the attitudes of the participants of the School Of General Practice (GP) Spring 2016 concerning ARP and compare the attitudes of the participants of the Alcohology course with participants from other courses. Material and Methods: We assessed the attitudes of the participants using the Short Alcohol and Alcohol Problems Perception Questionnaire (SAAPPQ). We investigated associations between sex, age, and workplace, and with results from SAAPPQ. Results: Two hundred and fifty eligible questionnaires were obtained. The average age was $28.1 \pm 2.2$ years old and $86.2 \%$ were female. The most represented workplace was Lisbon and Vale do Tejo. Pre-course, GPs' attitudes of both groups were homogeneous except on Adequacy and Role Security. The participants of the Alcohology course feel more secure in working with these patients. However, only $65 \%$ scored above midpoint in Therapeutic Commitment. Post-course, there were significantly higher values in all subscales for participants of the Alcohology course, with all scoring above midpoint in Safety and Therapeutic Commitment.

Discussion: The attitudes of the participants of the Alcohology course towards patients with ARP significantly improved after training in all evaluated subscales.

Conclusions: Courses that take into account not only GP's knowledge and skills, but also other attitudes, may be important to improve screening and treatment of patients with ARP. Explicit curricular attention to Self-esteem and Satisfaction may be required to improve further results.

Keywords: Alcohol; Attitudes; General practice; SAAPPQ; Training. 